\title{
List of Figures and Maps
}

\section{FIGURES}

I. Bailey Russel, View of Green River Lakes (2016) / 6

2. Bailey Russel, View of Flaming Gorge (2017) / 7

3. Kate Aitchison, Glen Canyon Dam (2016) / 8

4. Bailey Russel, View of Marble Canyon at Soap Creek (2018) / 9

5. Erika Osborne, Hoover Gates (20II) / io

6. Colorado River Basin Historical Water Supply and Use and Projected Future Water Supply and Demand (2012) / 83

7. David Jones, Subterranean BTUs (2010) / 99

8. Kate Aitchison, The Hull I (2017) / 100

9. Kate Aitchison, The Hull 2 (2017) / Ioo

Iо. Kate Aitchison, Katie Lee (2017) / IoI

I I. Erika Osborne, Looking for Moran (2OI2) / 102

I2. Brandon Gellis, Uncharted I-3 (2018) / 102

I3. Patrick Kikut, Powell Point (2018) / I04

I4. Chip Thomas, free yo mind + yo ass will follow (2013) / 195

I 5. Will Wilson, Auto Immune Response: Confluence of 3 Generations (2015) / 196

r6. Will Wilson, Melissa Pochoema, Insurgent Hopi Maiden (2015)/ 198 
$\mathrm{x} \quad$ Figures and Maps

I7. Chip Thomas, step in cow springs, navajo nation on the colorado plateau (2014) / I99

I8. Patrick Kikut, Dream Catcher (2017) / 200

MAPS

I. John Wesley Powell's Colorado River Exploring Expedition (I 869) / xxv

2. Dams and Diversions, Colorado River Basin (2019) / 5

3. Land Ownership, Colorado River Basin (2019) / 98

4. Native American Reservations, Colorado River Basin (2019) / I94

5. Distribution of Major Tribes in and around Colorado River Basin (c. I860) / 267

6. Major Military Posts and Transportation Networks in Southwest (I 846-60) / 268

7. Southwestern Reservations ( 1883 ) / 272 